\title{
Role of laparoscopic and robotic liver resection compared to open surgery in elderly hepatocellular carcinoma patients: a systematic review and meta- analysis
}

\author{
Alberto Brolese ${ }^{1}$, Marta Rigoni ${ }^{2,8}$, Alessandro Vitale ${ }^{3}$, Giovanni de Pretis ${ }^{4}$, Ivo Avancini ${ }^{4}$, Cecilia \\ Pravadelli ${ }^{4}$, Michela Frisinghelli ${ }^{5}$, Umberto Rozzanigo ${ }^{6}$, Giacomo Luppi ${ }^{6}$, Francesco Dionisi ${ }^{7}$, \\ Stefano Marcucci ${ }^{1}$, Giovanni Viel ${ }^{1}$, Paolo Beltempo ${ }^{1}$, Cristina Prezzi ${ }^{1}$, Marco Frisini ${ }^{1}$, Marco Brolese ${ }^{9}$, \\ Giandomenico Nollo2,8, Francesco A. Ciarleglio ${ }^{1}$ \\ 'Department of General Surgery \& HPB Unit (Chief: Alberto Brolese, MD), Azienda Provinciale per i Servizi Sanitari (APSS), \\ Santa Chiara Hospital, Trento 38122, Italy. \\ ${ }^{2}$ IRCS - Innovation and Clinical Health Research - Bruno Kessler Foundation (FBK), Trento 38123, Italy. \\ ${ }^{3}$ Department of Surgical, Oncological \& Gastroenterological Sciences, Padua University Hospital, Padua 35128, Italy. \\ ${ }^{4}$ Department of Hepatology \& Gastroenterology, APSS, Trento 38122, Italy. \\ ${ }^{5}$ Department of Oncology, Azienda Provinciale per i Servizi Sanitari (APSS), Trento 38122, Italy. \\ ${ }^{6}$ Department of Radiology, Azienda Provinciale per i Servizi Sanitari (APSS), Trento 38122, Italy. \\ ${ }^{7}$ Proton therapy Unit, Azienda Provinciale per i Servizi Sanitari (APSS), Trento 38122, Italy. \\ ${ }^{8}$ Department of Industrial Engineering, BIOtech Lab, University of Trento, Trento 38122, Italy. \\ ${ }^{9}$ School of Medicine, Padua University, Padua 35128, Italy.
}

Correspondence to: Dr. Alberto Brolese, Department of General Surgery \& HPB Unit, Azienda Provinciale per i Servizi Sanitari (APSS), Santa Chiara Hospital, Largo Medaglie d'oro, 1, Trento 38122, Italy. E-mail: alberto.brolese@apss.tn.it

How to cite this article: Brolese A, Rigoni M, Vitale A, de Pretis G, Avancini I, Pravadelli C, Frisinghelli M, Rozzanigo U, Luppi G, Dionisi F, Marcucci S, Viel G, Beltempo P, Prezzi C, Frisini M, Brolese M, Nollo G, Ciarleglio FA. Role of laparoscopic and robotic liver resection compared to open surgery in elderly hepatocellular carcinoma patients: a systematic review and meta-analysis. Hepatoma Res 2020;6:34. http://dx.doi.org/10.20517/2394-5079.2020.15

Received: 17 Feb 2020 First Decision: 11 Mar 2020 Revised: 27 Mar 2020 Accepted: 28 Apr 2020 Published: 18 Jun 2020

Science Editor: Bruno Nardo Copy Editor: Jing-Wen Zhang Production Editor: Tian Zhang

\begin{abstract}
Aim: This study aimed to compare mini-invasive liver resection (MILR) (laparoscopic/robotic approach) and open liver resection (OLR) for hepatocellular carcinoma $(H C C)$ in elderly patients with regard to clinical and oncological outcomes through a comprehensive systematic review.
\end{abstract}

Methods: The MEDLINE and Cochrane Library electronic databases were systematically searched from 2009 to December 2019 to identify relevant English written studies comparing MILR and OLR. The main endpoints were Child-Pugh score, serum total bilirubin level, comorbidity, presence/absence of cirrhosis, minor/major resection, challenge segment approach, operative time, estimated intraoperative blood loss, liver failure rate, morbidity according to the Clavien-Dindo classification, length of hospital stay (LOS), postoperative mortality, number of lesions, tumor size, readmission rate, recurrence rate and survival at 1, 3 and 5 years after operation. Meta-

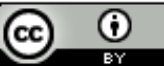

(c) The Author(s) 2020. Open Access This article is licensed under a Creative Commons Attribution 4.0 International License (https://creativecommons.org/licenses/by/4.0/), which permits unrestricted use sharing, adaptation, distribution and reproduction in any medium or format, for any purpose, even commercially, as long as you give appropriate credit to the original author(s) and the source, provide a link to the Creative Commons license, and indicate if changes were made.
}

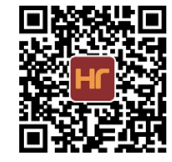


analyses provided pooled relative risks and mean differences for these outcomes. Cut-off for "elderly age" was set at 65 years old.

Results: Eight studies that evaluated 3051 patients who underwent liver resection for HCC, with 950 undergoing MILR and 2101 OLR, were included after the screening process. Blood loss, morbidity, and LOS showed statistical significance in favor of MILR. In particular, with respect to OLR, MILR decreased on average blood loss by $161.43 \mathrm{~mL}$ (95\% Cl: $250.24-72.61)$, risk of morbidity by $42 \%(P<0.01)$, LOS by 4 days $(95 \% \mathrm{Cl}: 7-2)$, postoperative mortality risk by $47 \%$ (although not significantly, $P=0.06$ ). Major resections were significantly more common in the OLR group $(P<0.0001)$. Recurrence, although not significant $(P=0.06)$, must also be emphasized. The two surgical approaches were comparable with regard to the other outcomes investigated.

Conclusion: Meta-analyses confirmed the advantages of MILR in terms of short perioperative outcomes, where it may promote the extension of liver resection to HCC patients with borderline liver function. MILR may be considered an important treatment option with significant benefits in the elderly and fragile patients. However, large well-designed prospective comparative studies or randomized controlled trials would be necessary to further confirm our conclusions.

Keywords: Hepatocellular carcinoma, HCC, mini-invasive liver resection, laparoscopic liver surgery, robotic liver surgery, open liver surgery, meta-analysis

\section{INTRODUCTION}

Hepatocellular carcinoma (HCC) is the most common primary liver neoplasm: it is the second leading cause of cancer-related deaths worldwide and sixth for cancer-related deaths in developed countries ${ }^{[1,2]}$. With regard to Italian data, HCC accounts for $79 \%$ of primary liver cancer, and it is among the first five causes of cancer-related deaths (7\% global population $)^{[3]}$.

The elderly rate in Italian and Western populations has increased for reduced newborn/year and the progressive increasing of mean age. The risk of developing cancer is age-dependent. In Italy, patients over 75 years old have a $25 \%$ higher relative risk than the 60-74 age group (147/100,000 vs. 106/100,000), and it is 5 times higher than the 45-59 age group ${ }^{[4]}$. In the next years, the true incidence of HCC will be directly related to population age up to rates of 51 cases/100,000 in males and 119 cases/100,000 in females ${ }^{[5]}$, according to EUROCARE report ${ }^{[6]}$. Therefore, the number of elderly patients requiring treatment for primary and metastatic liver cancer is constantly rising and, despite a limited life expectancy, the use of liver surgery has been found by many authors to be a safe and effective treatment for these patients ${ }^{[7,8]}$.

Laparoscopic liver resection for HCC in selected patients has shown very good results ${ }^{[9,10]}$ with regard to oncological outcomes, morbidity, mortality, length of hospital stay (LOS) and fast postoperative recovery. This is important after oncological surgery, because complications may negatively impact on short-term outcomes, long-term survival and recurrence ${ }^{[11]}$. The robotic approach has been introduced to overcome some limitations of conventional laparoscopy, such as improved range of movements and enhanced instrument dexterity, a 3-dimensional view of the surgical field, a reduction in surgeon tremors and shortened learning curve.

The effect of age on cancer treatment allocation is controversial ${ }^{[12]}$. Mini-invasive surgery is a new goal in the treatment of HCC because it has made a great impact on surgical practice and on liver surgery. The management of elderly patients with HCC is becoming routine in clinical practice, but it is substantially more complicated than with younger patients because of comorbidities such as cardiovascular and respiratory disease, diabetes, renal failure and fragility. Age may not represent a limiting factor for liver resection, but it is still unclear if elderly patients can benefit from minimally invasive surgery. The most common concerns 


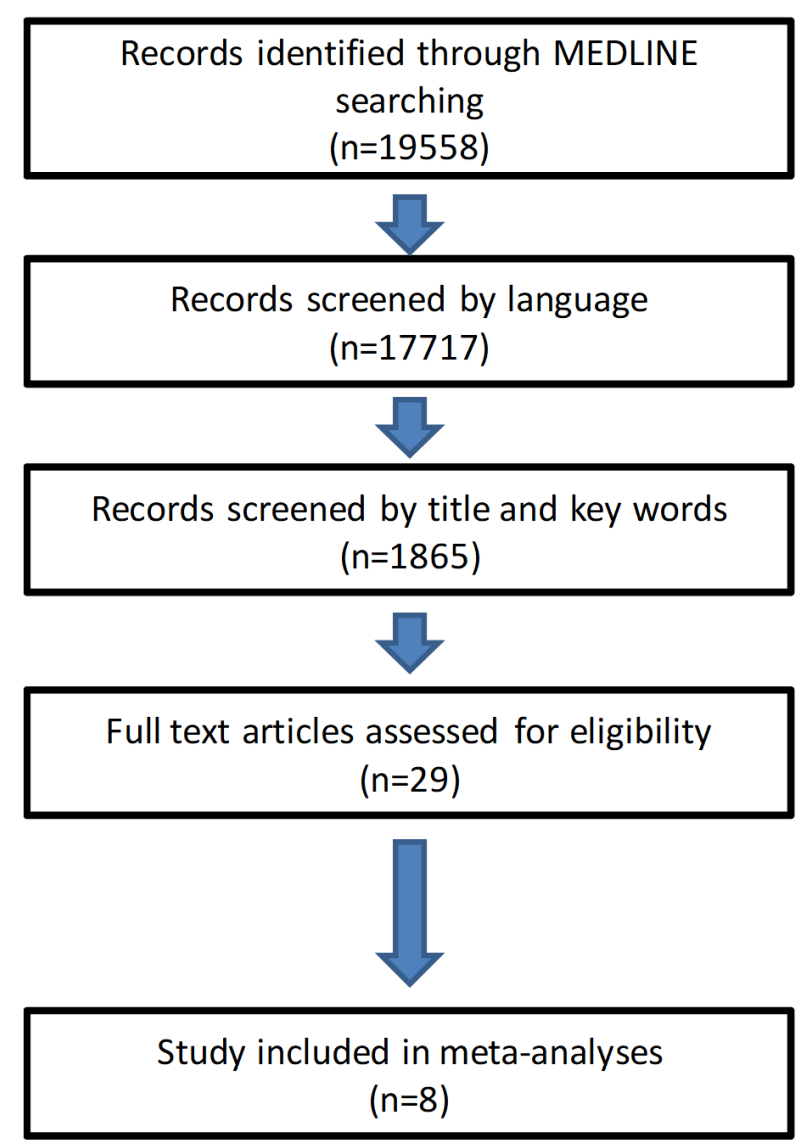

Population: el derly OR old age OR geriatrics population OR geriatric age AND hepatocellularcarcinoma ORHCCOR liver tumor OR Iiver cancer OR hepatoma Investigation/Comparison: liver resection OR liver surgery $O R$ open liver surgery OR open liver resection OR mini-invasive liver resection OR Iaparoscopic liver res ection OR robotic assisted liver resection OR mini-invasiveliver surgery OR laparoscopic liver surgery $O R$ robotic assisted liver surgery

Outcome: survival OR overall survival OR mortality OR morbidity OR recurrence $O R$ oncological outcomes OR blood loss OR length of stay OR LOS OR transfusion rate OR complications OR Dindo Clavien

Time: last ten years (2009-2019)

Types Articles: Clinical Trial, Comparative Study, Controlled Clinical Trial, Meta-Analysis, Multicenter Study, Observational Study, Randomized Controlled Trial

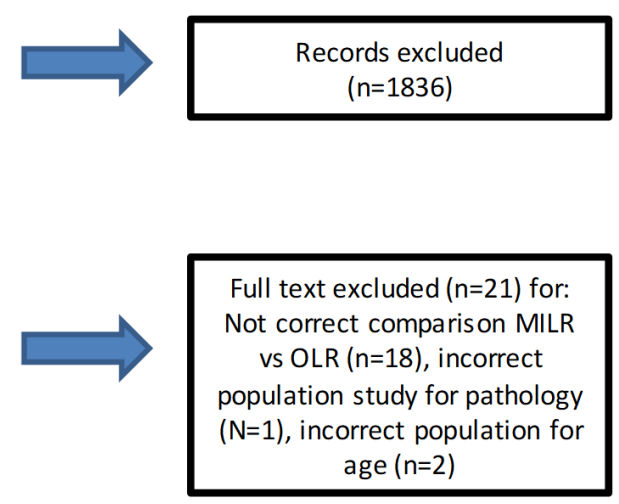

Figure 1. Flow chart of literature selection and PICOT description. MILR: mini-invasive liver resection; OLR: open liver resection

for surgeons and anesthesiologists in this regard are as follows: longer operative times, pneumoperitoneum and its physiological consequences, diminished functional reserve, and pre- or postoperative comorbidities.

In the last 10 years, only some single-center retrospective studies have analyzed the results of mini-invasive surgery in elderly patients, and very few reports have focused on topics about mini-invasive surgical treatment of the elderly with HCC.

The objective of this study was to perform a systematic review to compare mini-invasive liver resection (MILR) (laparoscopic/robotic approach) and open liver resection (OLR) for HCC in the elderly, across a comprehensive range of outcomes reported from both randomized and observational studies.

\section{METHODS}

\section{Literature search strategy}

Literature documenting a comparison of clinical and oncological outcomes in elderly patients who underwent MILR vs. OLR therapy for HCC was analyzed by searching PubMed and Cochrane Library from 2009 to December 2019. The search terms, either independently or in combination, were used according to PICOT framework [Figure 1]. A systematic search was conducted for relevant systematic reviews, randomized controlled trials, and observational studies (prospective or retrospective cohort and case-control or case-match studies) using a search strategy guided by oncological or surgical information, abstract and keywords related to our research question. Only English language published articles were screened. When more than one article was reported by the same institution or author, we selected either the one with the largest series or the most recent, with the exception of multicenter studies. 
The Preferred Reporting Items for Systematic Reviews and Meta-Analyses (PRISMA) statement checklist was used to report selection ${ }^{[13]}$.

\section{Study inclusion criteria}

In this section, we report the selection criteria to identify eligible studies for this review that aimed to compare studies on the effects of MILR vs. OLR for HCC in elderly patients. Different cut-offs for "elderly age" were considered in most studies. In the present study, the cut-off was set at 65 years old. Different resections, major or minor, were included. Study selection criteria were defined according to the PICOT framework [Figure 1]. Three different searches were identified: type 1, comparing open and laparoscopic liver resections; type 2, comparison in elderly between open and robotic liver resections; and type 3, comparison between laparoscopic and robotic liver resections. The following studies or data were excluded: case report, abstract, review, editorial letter, study without control group, and comparative study with population less than 10 patients for each group. The quality assessment of the primary studies did not represent exclusion criteria.

\section{Outcomes}

Primary outcomes of all eligible studies included Child-Pugh score, serum total bilirubin level, comorbidities, presence/absence of cirrhosis, minor/major resection, challenge segment approach, operative time, estimated intraoperative blood loss, liver failure rate, morbidity according to the Clavien-Dindo classification ${ }^{[14]}$, LOS, and postoperative mortality. Operative time was defined as the time from skin incision to wound closure. Postoperative mortality was defined as death during the same hospital admission or within 30 days after liver resection. Major resection was defined as a liver resection of three or more contiguous segments in all papers under investigation. Challenge segments were posterolateral segments (Sg6 and Sg7), posterosuperior segments (Sg8 and Sg4a) and caudate lobe. Secondary outcomes included tumor size, number of lesions (single/multiple), readmission rate, recurrence rate and survival at 1,3 and 5 years after operation.

\section{Data extraction and quality assessment}

Two reviewers (F.C. and M.R.) independently screened titles, abstracts, full texts, and extracted the demographic and clinical outcome data from the selected studies. When disagreement occurred, they reviewed the papers together to reach joint conclusions. The methodological quality of the studies was evaluated by applying the Critical Appraisal Skills Program - CASP Checklists for Case Control Study (Critical Appraisal Skills Programme (2018). CASP Case Control Study Checklist. Available at: https://caspuk.net/casp-tools-checklists/). The overall quality of the primary studies was assessed as low, moderate or high quality.

\section{Statistical analyses}

All the analyses were performed with the data originating from the included studies. When available, patient characteristics and outcomes were expressed as numbers or percentages, mean $\pm \mathrm{SD}$ or median (interquartile range or range), as reported in primary studies.

Some of the included studies reported the continuous variables with means and standard deviation, other studies with median and range or interquartile range. For continuous outcomes (i.e., operative time, estimated blood loss, and LOS), mean \pm SD for some primary studies were estimated from median, range, and interquartile range following the approach by Hozo et al. ${ }^{[15]}$ and Deeks et al. ${ }^{[16]}$. For mortality at 1, 3 and 5 years, row data (i.e., counts) were calculated by simple proportions by the given percentages of survivors in whole population of each primary study.

Eleven meta-analyses were performed, one for every outcome considered. A random effects model based on the method used by DerSimonian and Laird ${ }^{[17]}$ was used to estimate pooled risk ratios (RRs), pooled mean differences (MDs) and 95\% confidence intervals (95\%CIs). Data heterogeneity between studies was estimated 
Table 1. Characteristics and quality assessment of studies included in the systematic review

\begin{tabular}{|c|c|c|c|c|c|c|}
\hline Study & Year & Country & Study design & Surgical group & Number & Quality assessment ${ }^{\star \star}$ \\
\hline Badawy et al. ${ }^{[18]}$ & 2017 & Japan & Retro + PMS* & $\begin{array}{l}\text { MILR } \\
\text { OLR }\end{array}$ & $\begin{array}{l}40 \\
40\end{array}$ & $M$ \\
\hline Chan et al. ${ }^{[19]}$ & 2014 & China & Retro & $\begin{array}{l}\text { MILR } \\
\text { OLR }\end{array}$ & $\begin{array}{l}17 \\
34\end{array}$ & L \\
\hline Amato et al. ${ }^{[20]}$ & 2016 & Italy & Retro & $\begin{array}{l}\text { MILR } \\
\text { OLR }\end{array}$ & $\begin{array}{l}11 \\
18\end{array}$ & M \\
\hline Nomi et al. ${ }^{[21]}$ & 2020 & Japan & Retro-multicenter & $\begin{array}{l}\text { MILR } \\
\text { OLR }\end{array}$ & $\begin{array}{l}221 \\
409\end{array}$ & $\mathrm{H}$ \\
\hline Wang et al. ${ }^{[22]}$ & 2015 & China & Retro & $\begin{array}{l}\text { MILR } \\
\text { OLR }\end{array}$ & $\begin{array}{l}30 \\
60\end{array}$ & M \\
\hline Tee et al. ${ }^{[23]}$ & 2019 & USA & Retro-multicenter & $\begin{array}{l}\text { MILR } \\
\text { OLR }\end{array}$ & $\begin{array}{l}487 \\
1282\end{array}$ & $\mathrm{H}$ \\
\hline Wang et al. ${ }^{[24]}$ & 2018 & Taiwan & Retro & $\begin{array}{l}\text { MILR } \\
\text { OLR }\end{array}$ & $\begin{array}{l}63 \\
177\end{array}$ & M \\
\hline Chen et al. ${ }^{[25]}$ & 2017 & Taiwan & Retro + PMS* & $\begin{array}{l}\text { MILR } \\
\text { OLR }\end{array}$ & $\begin{array}{l}81 \\
81\end{array}$ & $\mathrm{H}$ \\
\hline
\end{tabular}

*PMS: propensity match score; ${ }^{\star \star}$ quality assessment evaluated by Critical Appraisal Skills Programme (2018). CASP Case Control Study Checklist (L: low quality; M: medium quality; H: high quality). MILR: mini-invasive liver resection; OLR: open liver resection

by Chi2, I2, and Tau2 statistics, which were determined by an inverse-variance fixed-effect model. Funnel plots graphically assessed publication bias.

A 2-tailed $P$ value $<0.05$ indicated statistical significance. All analyses were performed using the Cochrane Collaboration Software Review Manager 5 (version 5.2).

\section{RESULTS}

\section{Study characteristics and population}

The flow diagram for article selection for systematic review is shown in Figure 1 according to the PRISMA guidelines. The initial search yielded 19,558 reports but only 17,717 were in English. After examining the titles and key words, we excluded 15,852 citations because of irrelevance, and after abstract screening, we removed 1836 other records because of incongruences on population or outcomes. The 29 remaining studies were assessed for eligibility by a full-text examination. Finally, eight studies ${ }^{[18-25]}$ were included in this systematic review for the quantitative synthesis, five of which compared laparoscopic liver resection and open approach, two robotic $v s$. open liver resection and one both laparoscopic and robotic $v s$. open approach [Table 1].

A total of 3051 patients who underwent liver resection for HCC from 8 studies were included, with 950 undergoing MILR and 2101 OLR. All the selected studies were retrospective $(5$ case-control and 3 casematched). The cut-off age for elderly was 75 years old in 2 studies and 70 for 5 studies and median age was $>65$ for 1 study. Percentages of HCC patients were $100 \%$ for all the included studies with exception of Badawy et al. ${ }^{[18]}$ and Chan et al. ${ }^{[19]}$. For these two studies percentages of HCC patients in both mini-invasive and open groups were greater than $50 \%$, therefore we included the studies in the review and in the statistical analyses. The overall quality assessment of each of the studies included is given in Table 1. One study was assessed as low quality $^{[19]}$, four studies as moderate quality ${ }^{[18,20,22,24]}$ and three studies as high quality ${ }^{[21,23,25]}$.

\section{Primary outcomes}

Meta-analyses of the considered outcomes are reported in Figure 2A and B, Table 2 and Table 3. No significant differences in preoperative characteristics were noted between the groups for liver assessment and function, including Child-Pugh score, serum total bilirubin level, comorbidities, presence/absence of cirrhosis. Major resections were significantly more common in the OLR group compared to the MILR group; indeed, the relative risk for MILR was reduced by $42 \%(R R=0.58,95 \% C I$ : $0.34-0.97)$, but this result was affected by substantial heterogeneity $\left(I^{2}=86 \%\right)$. Segmentectomies and wedge resections were significantly 
A

Child-Plug score

Total bilirubin value (mg/dl)

Comorbidity

Presence/absence of Cirrhosis

Minor resections

Major resections

Challenge segments

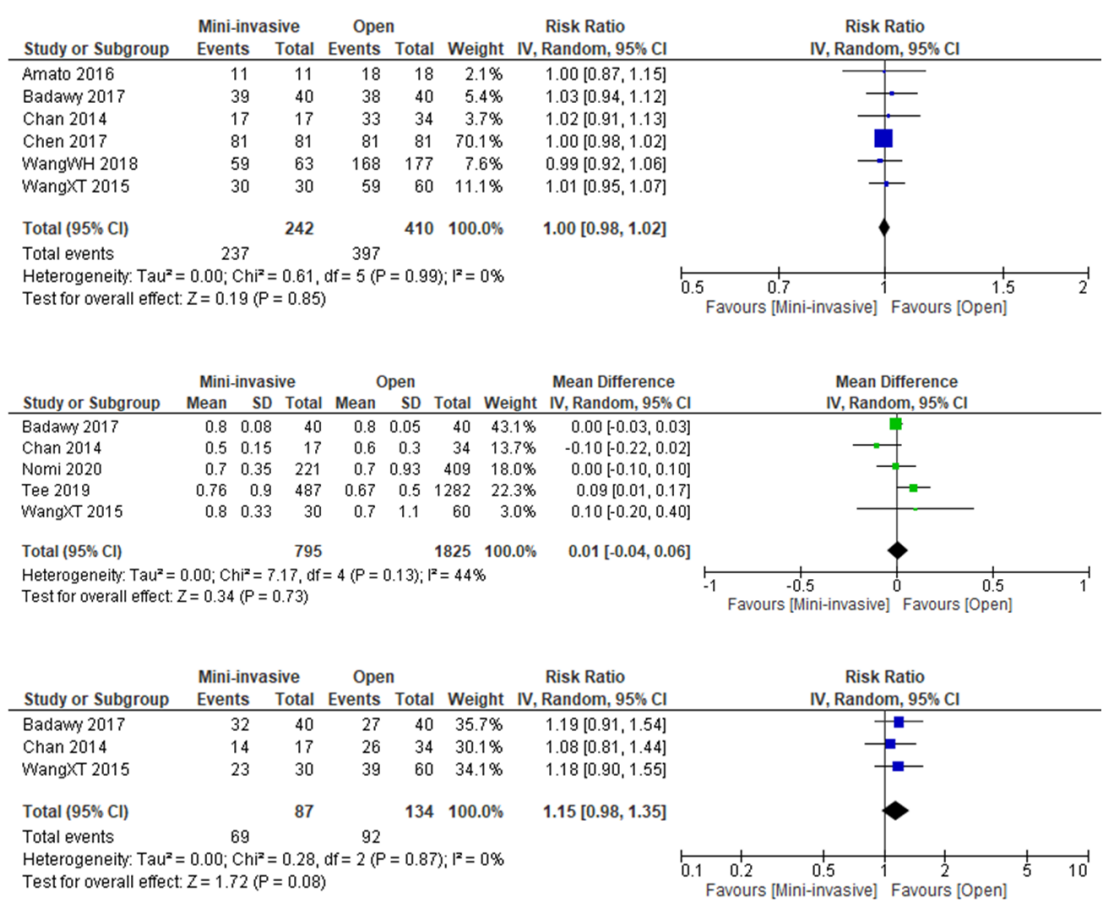

\begin{tabular}{|c|c|c|c|c|c|c|c|c|}
\hline \multirow[b]{2}{*}{ Study or Subgroup } & \multicolumn{2}{|c|}{ Mini-invasive } & \multicolumn{2}{|c|}{ Open } & \multicolumn{2}{|r|}{ Risk Ratio } & \multirow{2}{*}{\multicolumn{2}{|c|}{$\begin{array}{c}\text { Risk Ratio } \\
\text { IV, Random, } 95 \% \mathrm{Cl}\end{array}$}} \\
\hline & Events & Total & Events & Total & Weight & IV, Random, $95 \% \mathrm{Cl}$ & & \\
\hline Badawy 2017 & 7 & 40 & 13 & 40 & $9.7 \%$ & $0.54[0.24,1.21]$ & & \\
\hline Chan 2014 & 9 & 17 & 11 & 34 & $13.6 \%$ & $1.64[0.84,3.17]$ & & \\
\hline Chen 2017 & 37 & 81 & 38 & 81 & $34.4 \%$ & $0.97[0.70,1.36]$ & & \\
\hline WangWH 2018 & 15 & 63 & 53 & 177 & $21.0 \%$ & $0.80[0.48,1.31]$ & & \\
\hline Wangx<T 2015 & 14 & 30 & 24 & 60 & $21.2 \%$ & $1.17[0.71,1.91]$ & & \\
\hline Total $(95 \% \mathrm{Cl})$ & & 231 & & 392 & $100.0 \%$ & $0.98[0.75,1.29]$ & & \\
\hline Total events & 82 & & 139 & & & & & \\
\hline $\begin{array}{l}\text { Heterogeneity. Tau } \\
\text { Test for overall effec }\end{array}$ & $\begin{array}{l}0.03 ; \mathrm{Chi}^{2} \\
Z=0.13(\mathrm{~F}\end{array}$ & $\begin{array}{l}=5.59, \\
=0.90\end{array}$ & $d f=4(P=$ & $=0.23)$ & $1_{12}=28 \%$ & & $\begin{array}{ccc}0.01 & 0.1 & 1 \\
\text { Favours [Mini-invasive] } & \\
& \text { Favours [0 }\end{array}$ & {$\left[\begin{array}{c}10 \\
\text { [Open] }\end{array}\right.$} \\
\hline
\end{tabular}

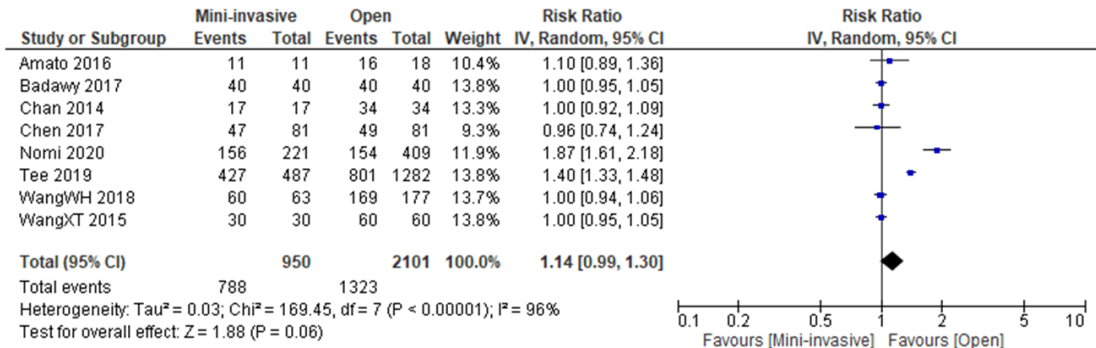

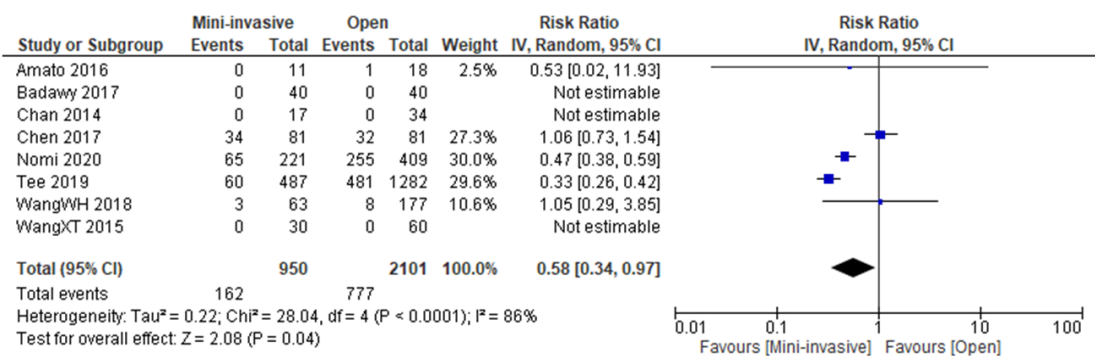

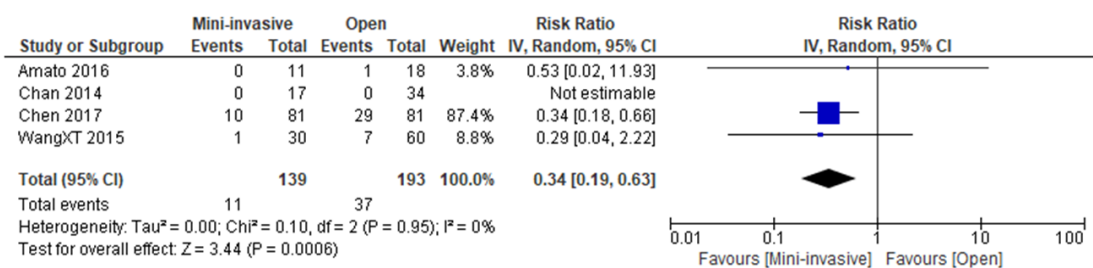


B

\section{Operative time}

\section{Blood loss}

Liver failure rate

\section{Clavien-Dindo morbidity}

\section{Length of hospital stay (LOS)} mortality
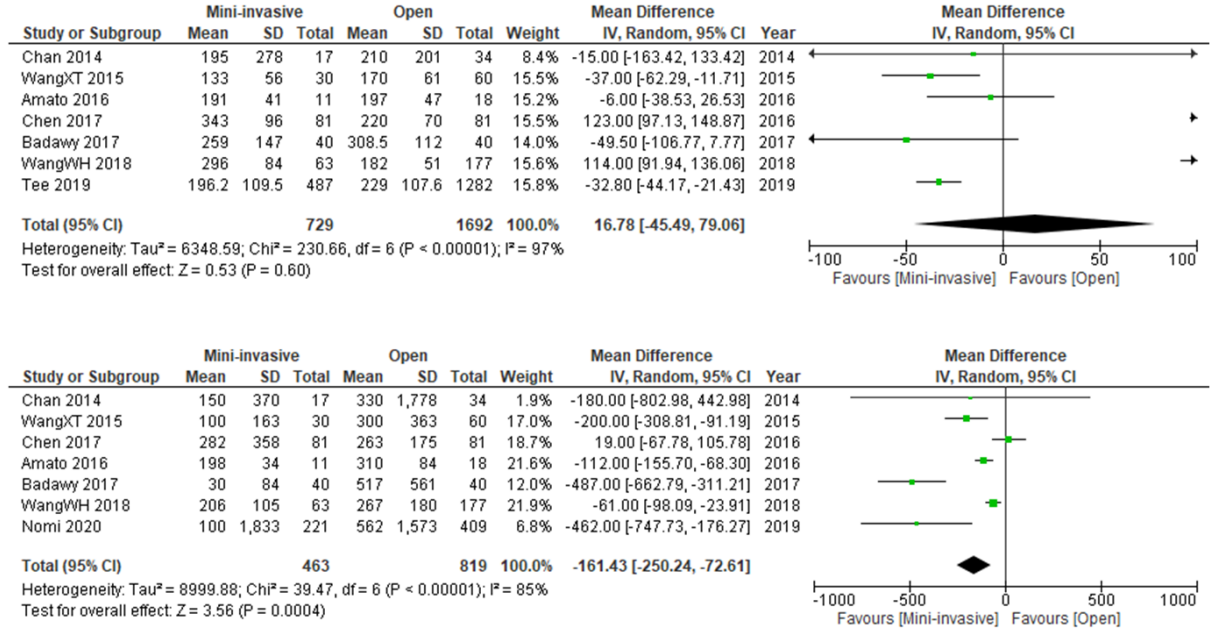
Test for overall effect $Z=3.56(P=0.0004)$

\begin{tabular}{|c|c|c|c|c|c|c|c|c|c|c|c|}
\hline \multirow[b]{2}{*}{ Study or Subgroup } & \multicolumn{2}{|c|}{ Mini-invasive } & \multicolumn{2}{|c|}{ Open } & \multicolumn{3}{|c|}{ Risk Ratio } & \multirow{2}{*}{\multicolumn{4}{|c|}{$\begin{array}{c}\text { Risk Ratio } \\
\text { IV, Random, } 95 \% \mathrm{CI}\end{array}$}} \\
\hline & Events & Total & Events & Total & Weight & IV, Random, $95 \% \mathrm{Cl}$ & Year & & & & \\
\hline Chan 2014 & 0 & 17 & 0 & 34 & & Not estimable & 2014 & & & & \\
\hline Chen 2017 & 0 & 81 & 0 & 81 & & Not estimable & 2016 & & & & \\
\hline Amato 2016 & 0 & 11 & 0 & 18 & & Not estimable & 2016 & & & & \\
\hline Badawy 2017 & 5 & 40 & 11 & 40 & $27.2 \%$ & $0.45[0.17,1.19]$ & 2017 & & & & \\
\hline WangWH 2018 & 0 & 63 & 1 & 117 & $19.2 \%$ & $0.61[0.03,14.87]$ & 2018 & & & & \\
\hline Nomi 2020 & 7 & 221 & 36 & 409 & $27.6 \%$ & $0.36[0.16,0.80]$ & 2019 & $\longrightarrow$ & & & \\
\hline Tee 2019 & 62 & 487 & 2 & 1282 & $26.0 \%$ & $81.61[20.04,332.32]$ & 2019 & & & & 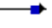 \\
\hline Total $(95 \% \mathrm{Cl})$ & & 920 & & 1981 & $100.0 \%$ & $1.74[0.15,20.18]$ & & & & & \\
\hline Total events & 74 & & 50 & & & & & & & & \\
\hline $\begin{array}{l}\text { Heterogeneity. } \mathrm{Tau}^{2}= \\
\text { Test for owerall effect }\end{array}$ & $\begin{array}{l}5.50 ; \mathrm{Chi}^{-} \\
\mathrm{z}=0.44(\end{array}$ & $\begin{array}{l}=46.70 \\
=0.66\end{array}$ & & & $0001) ; 1^{2}=$ & & & 0.01 & & 10 & $100^{\circ}$ \\
\hline
\end{tabular}

Mini-invasive Open Risk Ratio

Study or Subgroup Events Total Events Total Weight IV, Random, $95 \% \mathrm{Cl}$ Year

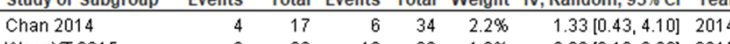
Chan 2014 Chen 2017 Amato 2016 Badawy 2017 WangWH 2018 Nomi 2020 Tee 2019

Total $(95 \% \mathrm{Cl})$ Total events Heterogeneity: $\operatorname{Tau}^{2}=0.00 ; \mathrm{Ch}^{2}=6.28, \mathrm{df}=7(\mathrm{P}=0.51) ; \mathrm{I}^{2}=0 \%$ Test for overall effect $z=6.38(P=0.00001)$ $1.33[0.43,4.10] 2014$ $0.60[0.18,2.02] 2015$
$1.00[0.26,3.86] 2016$ $1.00[0.26,3.86] 2016$ $0.12[0.01,1.97] 2016$ $\begin{array}{ll}0.40[0.17,0.93] & 2017 \\ 0.73[0.33,1.59] & 2018\end{array}$ $0.50[0.37,0.68] 201$ $0.62[0.50,0.77] 2019$ $0.58[0.50,0.69]$

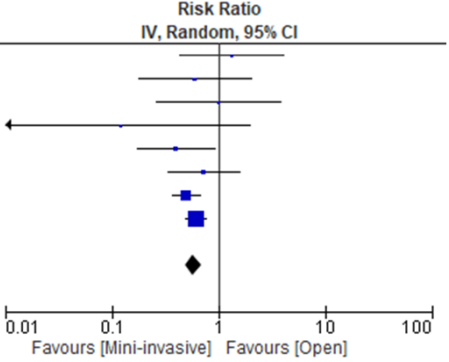

Mini-inyasive Open Mean Difference

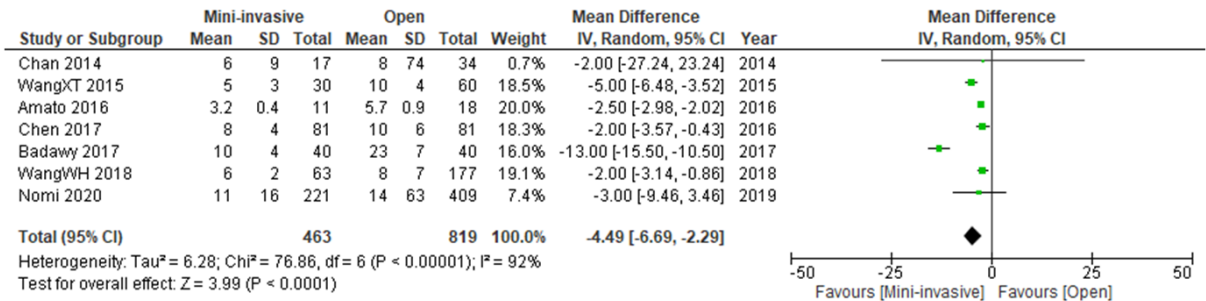
Test for overall effect: $Z=3.99(P<0.0001)$

Risk Ratio

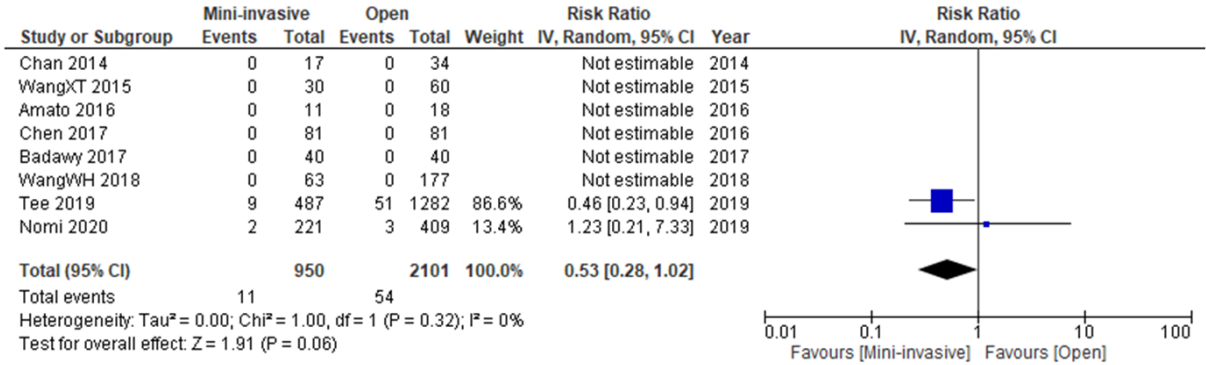

Figure 2. A: Meta-analyses of included studies on primary outcomes; B: Meta-analyses of included studies on primary outcomes 
Table 2. Clinical characteristics and primary outcomes of studies included in the meta-analysis

\begin{tabular}{|c|c|c|c|c|c|c|c|c|c|c|c|c|c|}
\hline \multirow[t]{2}{*}{ Study } & \multirow[t]{2}{*}{ Year } & \multicolumn{2}{|c|}{ CP score $A$} & \multicolumn{2}{|c|}{$\begin{array}{c}\text { Mean total } \\
\text { bilirubin }(\mathrm{mg} / \mathrm{dL})\end{array}$} & \multicolumn{2}{|c|}{$\begin{array}{c}\text { Comorbidity } \\
\text { (pathological history) }\end{array}$} & \multicolumn{2}{|c|}{$\begin{array}{l}\text { Cirrhosis } \\
\text { (presence) }\end{array}$} & \multicolumn{2}{|c|}{$\begin{array}{l}\text { Minor } \\
\text { resection }\end{array}$} & \multicolumn{2}{|c|}{$\begin{array}{c}\text { Major } \\
\text { resection }\end{array}$} \\
\hline & & MILR & OLR & MILR & OLR & MILR & OLR & MILR & OLR & MILR & OLR & MILR & OLR \\
\hline Badawy et al. ${ }^{[18]}$ & 2017 & $39 / 40$ & $38 / 40$ & 0.8 & $7 / 40$ & $40 / 40$ & $40 / 40$ & $40 / 40$ & $40 / 40$ & $6 / 40$ & $6 / 40$ & 22 & 23.5 \\
\hline Chan et al. ${ }^{[19]}$ & 2014 & $17 / 17$ & $33 / 34$ & 0.5 & $9 / 17$ & $17 / 17$ & $34 / 34$ & $17 / 17$ & $34 / 34$ & $3 / 17$ & $12 / 34$ & 30 & 30 \\
\hline Amato et al. ${ }^{[20]}$ & 2016 & $11 / 11$ & $18 / 18$ & na & $11 / 11$ & $11 / 11$ & $16 / 18$ & $11 / 11$ & $16 / 18$ & 0 & 0 & 35.45 & 39.83 \\
\hline Nomi et al. ${ }^{[21]}$ & 2020 & na & na & 0.7 & na & $156 / 221$ & $154 / 409$ & $156 / 221$ & $154 / 409$ & na & na & 25 & 38 \\
\hline Wang et al. ${ }^{[22]}$ & 2015 & $30 / 30$ & $59 / 60$ & 0.8 & $14 / 30$ & $30 / 30$ & $60 / 60$ & $30 / 30$ & $60 / 60$ & $3 / 30$ & $7 / 60$ & 40 & 50 \\
\hline Tee et al. ${ }^{[23]}$ & 2019 & na & na & 0.76 & na & $427 / 487$ & $801 / 1282$ & $427 / 487$ & $801 / 1282$ & na & na & na & na \\
\hline Wang et al. ${ }^{[24]}$ & 2018 & $59 / 63$ & $168 / 177$ & na & $15 / 63$ & $60 / 63$ & $169 / 177$ & $60 / 63$ & $169 / 177$ & $8 / 63$ & $35 / 177$ & 36.3 & 30.5 \\
\hline Chen et al. ${ }^{[25]}$ & 2017 & $81 / 81$ & $81 / 81$ & na & $37 / 81$ & $47 / 81$ & $49 / 81$ & $47 / 81$ & $49 / 81$ & na & na & na & na \\
\hline
\end{tabular}

MILR: mini-invasive liver resection; OLR: open liver resection; CP: Child-Pugh

Table 3. Further primary outcomes of studies included in the meta-analysis

\begin{tabular}{|c|c|c|c|c|c|c|c|c|c|c|c|c|c|c|c|}
\hline \multirow[t]{2}{*}{ Study } & \multirow[t]{2}{*}{ Year } & \multicolumn{2}{|c|}{$\begin{array}{c}\text { Challenge segment } \\
\text { resection }\end{array}$} & \multicolumn{2}{|c|}{$\begin{array}{l}\text { Operative } \\
\text { time (min) }\end{array}$} & \multicolumn{2}{|c|}{ Liver failure } & \multicolumn{2}{|c|}{$\begin{array}{l}\text { Blood loss } \\
(\mathrm{mL})\end{array}$} & \multicolumn{2}{|c|}{ Morbidity } & \multicolumn{2}{|c|}{ Mortality } & \multicolumn{2}{|c|}{$\begin{array}{l}\text { LOS } \\
\text { (Day) }\end{array}$} \\
\hline & & MILR & OLR & MILR & OLR & MILR & OLR & MILR & OLR & MILR & OLR & MILR & OLR & MILR & OLR \\
\hline Badawy et al. ${ }^{[18]}$ & 2017 & na & na & 259 & 308.5 & $5 / 40$ & $11 / 40$ & 30 & 517 & $6 / 40$ & $15 / 40$ & $0 / 40$ & $0 / 40$ & 10 & 23 \\
\hline Chan et al. ${ }^{[19]}$ & 2014 & $0 / 17$ & $0 / 34$ & 195 & 210 & $0 / 17$ & $0 / 34$ & 150 & 330 & $4 / 17$ & $6 / 34$ & $0 / 17$ & $0 / 34$ & 6 & 8 \\
\hline Amato et al. ${ }^{[20]}$ & 2016 & $0 / 11$ & $1 / 18$ & 190.9 & 196.9 & $0 / 11$ & $0 / 18$ & 198 & 310 & $0 / 11$ & $6 / 18$ & $0 / 11$ & $0 / 18$ & 3.18 & 5.7 \\
\hline Nomi et al. ${ }^{[21]}$ & 2020 & na & na & na & na & $7 / 221$ & $36 / 409$ & 100 & 562 & $40 / 221$ & $149 / 409$ & $2 / 221$ & $3 / 409$ & 11 & 14 \\
\hline Wang et al. ${ }^{[22]}$ & 2015 & $1 / 30$ & $7 / 60$ & 133 & 170 & na & na & 100 & 300 & $3 / 30$ & $10 / 60$ & $0 / 30$ & $0 / 60$ & 5 & 10 \\
\hline Tee et al. ${ }^{[23]}$ & 2019 & na & na & 196.2 & 229.0 & $62 / 487$ & $2 / 1282$ & na & na & $81 / 487$ & $346 / 1282$ & $9 / 487$ & $51 / 1282$ & na & na \\
\hline Wang et al. ${ }^{[24]}$ & 2018 & na & na & 296 & 182 & $0 / 63$ & $1 / 177$ & 206 & 267 & $7 / 63$ & $27 / 177$ & $0 / 63$ & $0 / 177$ & 6.21 & 8.18 \\
\hline Chen et al. ${ }^{[25]}$ & 2017 & $10 / 81$ & $29 / 81$ & 343 & 220 & $0 / 81$ & $0 / 81$ & 282 & 263 & $4 / 81$ & 4/81 & $0 / 81$ & $0 / 81$ & 7.5 & 10.1 \\
\hline
\end{tabular}

MILR: mini-invasive liver resection; LOS: length of hospital stay; OLR: open liver resection

more common in the MILR than in the OLR group ( $\mathrm{RR}=0.34,95 \% \mathrm{CI}$ : 0.19-0.63). The risk for the miniinvasive group with respect to the open group was reduced by $66 \%$, but the result showed considerable heterogeneity $\left(I^{2}=95 \%\right)$ and these results were however related to only one study ${ }^{[25]}$.

Among the thirteen outcomes, estimated blood loss, morbidity according to Clavien-Dindo classification, and LOS showed statistical significance in favor of the mini-invasive approach. In particular, on average, mini-invasive intervention decreased blood loss by 161.43 (95\%CI: 250.24$72.61) \mathrm{mL}$, although this result showed a substantial percentage of statistical heterogeneity $\left(I^{2}=85 \%\right)$ between studies. The mini-invasive approach decreased the risk of morbidity by $42 \%$ with respect to open resection $(P<0.01)$, and these pooled data were strengthened by no important heterogeneity between studies $\left(I^{2}=0 \%\right)$. LOS indicated an average decrease of 4 (95\%CI: 7-2) days for mini-invasive with respect to open surgery, even if this effect showed considerable heterogeneity $\left(I^{2}=92 \%\right)$ between studies. Finally, postoperative mortality showed a risk reduction of $47 \%$ for mini-invasive compared to open surgery, although not significant $(P=0.06)$. Due to zero events both in the mini-invasive and open groups, 6 out of 8 studies were not informative for this outcome. Consequently, this outcome was estimated by 2 out of 8 studies, that demonstrated no important statistical heterogeneity $\left(I^{2}=0 \%\right)$. Funnel plots of each outcome showed no graphical asymmetry, indicating no publication bias, although the number of studies was too low to support strong deductions.

\section{Secondary outcomes}

Meta-analyses of the outcomes considered are shown in Figure 3. Number of lesions (single/multiple), readmission rate, recurrence rate, survival at 1,3 , and 5 years showed no statistical differences between the mini-invasive and open groups. Tumor size plotting analysis reported a mean pooled size reduction of $4.22 \mathrm{~mm}$ in the MILR group, although this result was not statistically significant (95\%CI: 9.57-1.13, $P=0.12$ ), and heterogeneity was substantial $\left(I^{2}=84 \%\right)$. In particular, the recurrence outcome, estimated by two studies, 
Tumor size $(\mathrm{mm})$

Patients with multiple lesions

\section{Readmission}

Recurrence

Survival at 1 year

Survival at 3 years

\section{Survival at 5 years}

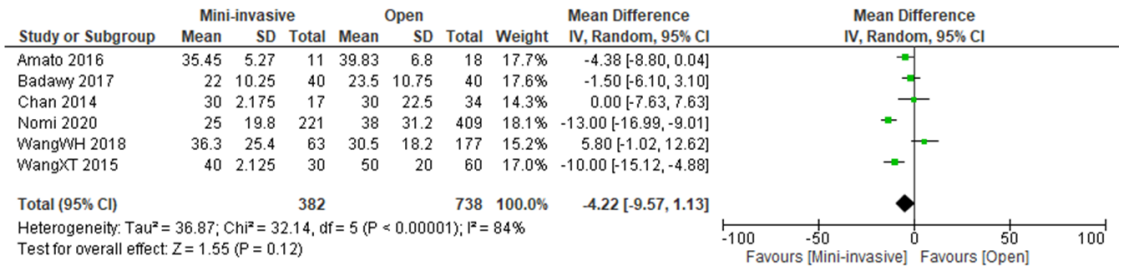

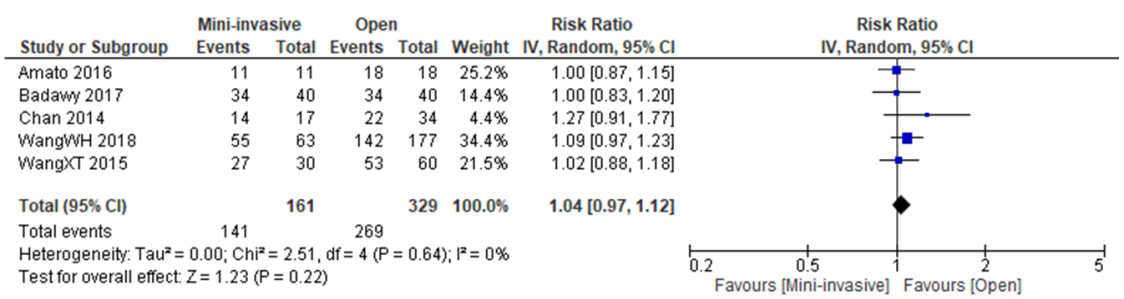

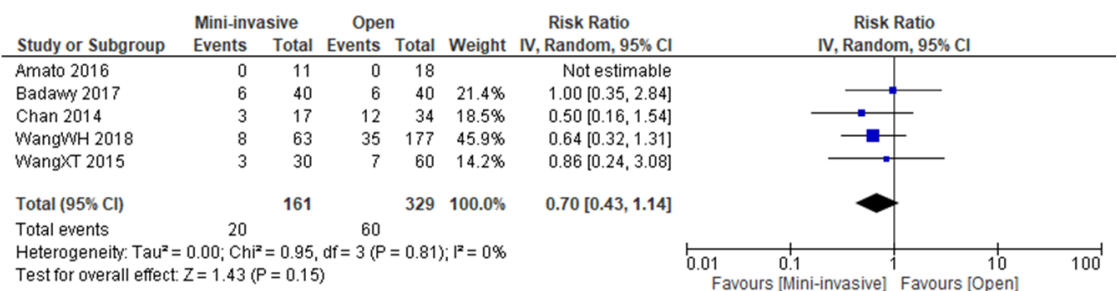

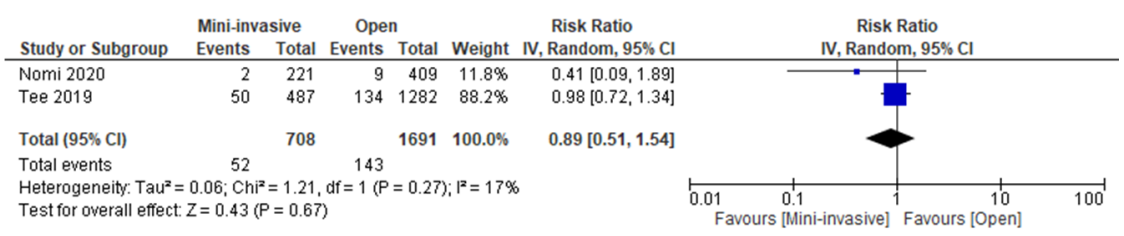
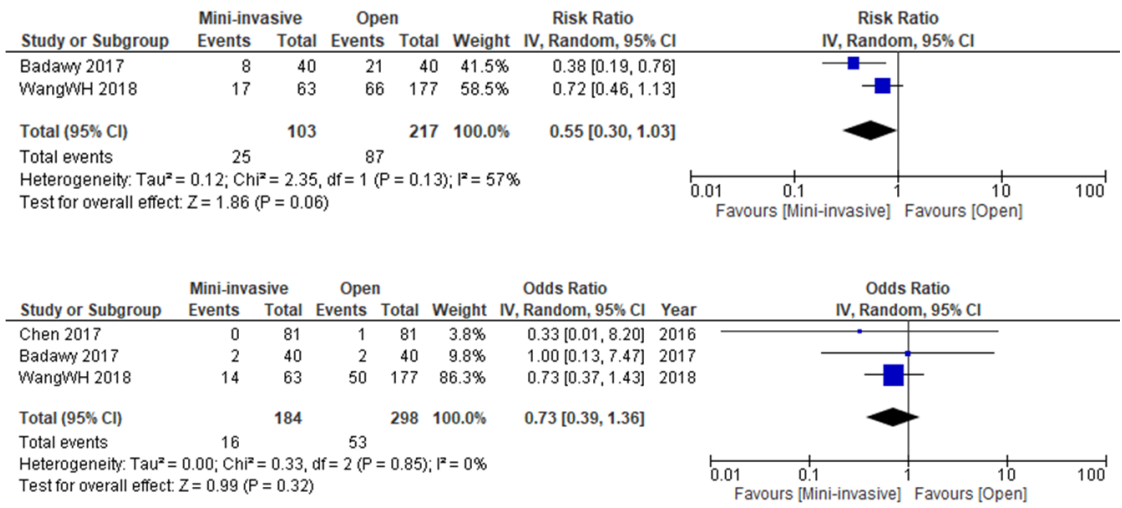

Test for overall effect $Z=0.99(P=0.32)$

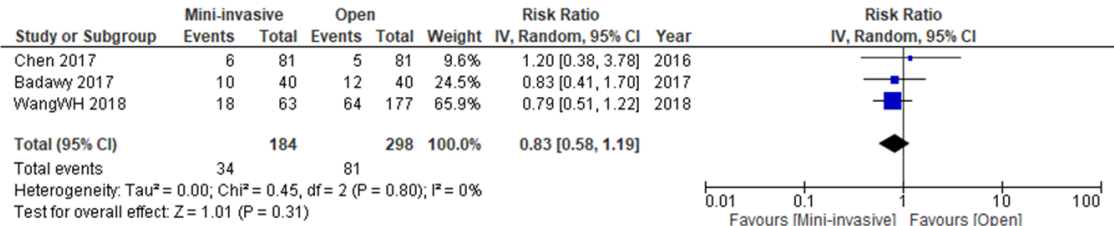

Test for overall effect: $Z=1.01(P=0.31)$

\begin{tabular}{|c|c|c|c|c|c|c|c|c|c|}
\hline \multirow[b]{2}{*}{ Study or Subgroup } & \multicolumn{2}{|c|}{ Mini-invasive } & \multicolumn{2}{|c|}{ Open } & \multicolumn{3}{|c|}{ Risk Ratio } & \multirow{2}{*}{\multicolumn{2}{|c|}{$\begin{array}{c}\text { Risk Ratio } \\
\text { IV, Random, } 95 \% \mathrm{Cl}\end{array}$}} \\
\hline & Events & Total & Events & Total & Weight & IV, Random, $95 \% \mathrm{Cl}$ & Year & & \\
\hline Badaw 2017 & 21 & 40 & 21 & 40 & $51.7 \%$ & $1.00[0.66,1.52]$ & 2017 & & \\
\hline WangWH 2018 & 18 & 63 & 69 & 177 & $48.3 \%$ & $0.73[0.48,1.13]$ & 2018 & & \\
\hline Total $(95 \% \mathrm{Cl})$ & & 103 & & 217 & $100.0 \%$ & $0.86[0.63,1.17]$ & & & \\
\hline Total events & 39 & & 90 & & & & & & \\
\hline $\begin{array}{l}\text { Heterogeneity: } \mathrm{Tau}^{2} \\
\text { Test for overall effect }\end{array}$ & $\begin{array}{l}0.00 ; \mathrm{Ch} \\
\mathrm{z}=0.97\end{array}$ & $\begin{array}{l}=1.03, \\
=0.33\end{array}$ & & $=0.31$ & $1) ; 1^{2}=3 \%$ & & & 0.01 & 100 \\
\hline
\end{tabular}

Figure 3. Meta-analyses of included studies on secondary outcomes 
demonstrated a risk reduction of $45 \%$ for the mini-invasive group with respect to the open one, although not significant $(P=0.06)$ and with moderate heterogeneity $\left(I^{2}=57 \%\right)$. However, the number of studies for each outcome was too low to evaluate publication bias.

\section{DISCUSSION}

The management of HCC in elderly patients is multidisciplinary with a wide range of treatment options ranging from liver resection, liver transplantation, loco-regional therapies including ablation and transarterial-chemoembolization, to molecular-targeting therapies ${ }^{[26]}$. The right patient allocation is determined by many factors including clinical characteristics, tumor burden, and multidisciplinary staff expertis ${ }^{[27]}$. Elderly patients have increased comorbidities including cardiovascular disease, pulmonary disease, diabetes mellitus, and renal insufficiency: these are conditional factors for outcome after surgical therapy as compared to the younger population ${ }^{[28]}$. Mini-invasive liver surgery represents a particular challenge for elderly patients affected by cardiopulmonary disease. Carbon dioxide pneumoperitoneum may result in acid-base disturbance with acidosis ${ }^{[29]}$ and the increase of intra-abdominal pressure may result in a decrease in lung compliance, vital capacity, venous return and vascular perfusion of intra-abdominal $\operatorname{organs}^{[30]}$.

In the last 10 years, improvement of perioperative care, careful patient selection and the presence of strong clinical evidence of benefits have increased the application of laparoscopic procedures in elderly patients. Several studies have reported on the safety and reduced postoperative morbidity and mortality in laparoscopic surgery in elderly patients ${ }^{[31,32]}$. Randomized trials, multicenter trials, systematic reviews, and meta-analyses about laparoscopic colorectal resection in the elderly indicate a real benefit in terms of lower risk of blood transfusion, postoperative complications and oncological outcome. Longer operative time and pneumoperitoneum seem to promote short-term pulmonary and/or cardiac complications ${ }^{[33-36]}$.

Surgical resection is a potentially curative option for the elderly patient. Several meta-analyses ${ }^{[37,38]}$ have shown that laparoscopic and robotic liver resection is associated with faster recovery, less postoperative pain and shorter hospital stay when compared with open liver resection.

Although the elderly could have a more complex clinical profile and a number of fragilities, age is not an absolute contraindication to liver surgery. The Barcelona Clinic Liver Cancer staging and treatment algorithm recommend surgical resection as elective treatment without difference between young or elderly $^{[39]}$. Nevertheless, the correct determination of which patients in the elderly group would benefit from surgical therapy is the most important clinical challenge. Poor liver function, portal hypertension, important comorbidities and cirrhosis stage are the true selection criteria for the right therapy and are helpful for identifying unfit patients.

Many studies have already demonstrated the feasibility of liver resections by the open approach in elderly patients including those suffering from other concomitant diseases ${ }^{[40]}$, but the role of the mini-invasive approach (laparoscopic or robotic) in the surgical management of HCC is under investigation.

This systematic review focused on the elderly population affected by HCC to assess if MILR may be safe and feasible in this group of fragile patients. In this study, we included eight primary studies with a total of 3051 patients undergoing liver resection; 950 were treated by MILR and 2101 by OLR. Using these data, we performed twenty-one meta-analyses investigating the main clinical and oncological outcomes of relevance. Regarding the functional selection criteria for MILR or OLR in HCC patients, all papers ${ }^{[18-25]}$ reported that the only patients considered eligible were those with well-compensated cirrhosis or liver function without severe portal hypertension or bilirubin level out of normal range. They were essentially identical in both groups (OLR and MILR), because a careful patient selection and a complete liver function assessment were mandatory in these patients. Of all meta-analyses investigated, only 8 patients in the OLR group ${ }^{[18,19,22,24]}$ and only 2 
patients in the MILR group ${ }^{[18,24]}$ were identified as Child-Pugh score B. These data, as shown in Figure 2A, however represented a study limitation since the present meta-analysis was not able to find statistically significant results.

Meta-analyses demonstrated that in elderly patients MIRL had similar organ failure, mortality and readmission rate as compared to the open approach. MILR can be safe in the elderly because it requires less sacrifice of liver tissue and has better bleeding control and lower rate of intermittent Pringle maneuver and because it can treat multiple lesions at the same time, especially in anterior segments. However, there are cases where complete MILR is not possible and use of ablative therapy combined with surgery increase oncological outcome $e^{[41]}$.

Nomi et al. ${ }^{[21]}$ demonstrated in their series that MILR was safer and more feasible when compared with OLR, even in octogenarian patients. This study was the first multicenter, propensity score-matched study to show better short-term outcomes with MILR than with OLR in elderly patients with HCC. These authors performed a subgroup analysis according to patient's age (group 75-79 compared with group $>80$ ) and dividing patients in relation to treatment (MILR - 78 patients and OLR -147 patients). In the cohort $>80$, the major complication rate and LOS were significantly lower in the MILR group than OLR group. Furthermore, in the MILR group, the study reported both a 90 -day mortality rate and transfer to rehabilitation facility rate of $0 \%$ in the MILR group. These data suggested that mini-invasive surgery was less invasive and was associated with early recovery in elderly patients.

In our analysis, morbidity rate according to the Clavien-Dindo classification, LOS and intraoperative blood loss were lower in the mini-invasive group with high statistical impact. These findings were consistent with many studies and meta-analyses on major resection ${ }^{[42-44]}$. The Southampton Guidelines reported that the laparoscopic approach was found to be the only independent factor to reduce the complication rate in resections for $\mathrm{HCC}^{[45]}$. In cirrhotic patients, the laparoscopic approach reduces the incidence of postoperative ascites, liver failure and morbidity assessed in terms of "Comprehensive Complication Index" ${ }^{\text {"[4-47] }}$. Blood loss and transfusion rate are very important prognostic factors in liver surgery ${ }^{[48]}$. Morbidity rate reduction demonstrated by our metaanalysis in patients undergoing MIRL could be explained by many factors. First, pneumoperitoneum with abdominal negative pressure decreased portal flow rate and reduced the small and continuous venous bleeding during the parenchymal transection phase ${ }^{[49]}$. Second, the use of an energy instrument for transection of liver parenchyma has proved to be highly effective for hemostasis ${ }^{[50]}$. Moreover, the absence of a large abdominal skin incision reduces muscle wall bleeding, and finally, laparoscopy and robotic technology offer an optimal magnified and three-dimensional view, which are important surgical factors for meticulous hemostasis as well as for greatly facilitating parenchymal transection in cirrhotic livers ${ }^{[51]}$.

However, one of the major limitations of our meta-analysis could be that surgical indications to MILR were selected at the center's discretion according to surgical procedure complexity rather than by defined criteria. All authors included in this meta-analysis always reported the principles guiding patient selection to undergo MIRL were according to the International Position on Mini-Invasive Liver Surgery agreement of Louisville (2008) or Morioka Guidelines $(2014)^{[9,10,24]}$, tumor size ${ }^{[24]}$ and tumor location ${ }^{[18,19,20,22,24]}$. An important point that needs to be investigated is that all papers reported many minor liver resections in the MILR group rather than in the OLR group. However, it remains uncertain if the same short and long benefits could be extended to elderly patients with major anatomical resection involving larger parenchymal transection area or longer operative time. Wang et al. ${ }^{[24]}$ found in their study that $38 \%$ of HCC cases in the robotic assisted group were located in challenge segments, but they never performed a major hepatectomy in the MILR group. The large number of minor resections, wedge or segmentectomies, suggested that a parenchymal sparing strategy and Ro resections are however basic and main guidelines for treatment when using a mini-invasive technique. This means that the mini-invasive cohort included in this paper was certainly not previously highly selected because all authors, as stated, followed international guidelines. 
Amato et al ${ }^{[20]}$ wrote that the main factor that would contribute to decreased blood loss might be the tumor position in anterior segments. Challenge segment resection in their series was performed only with the open approach. This selection might have had significant effects in reducing severe bleeding risks, but the robotic approach can represent the ideal overlap technique to overcome the bias in their study ${ }^{[52]}$.

Pulmonary and cardiovascular failure after liver resection might be very dangerous in the elderly. The incidence range has been reported to be from $10 \%$ to $20 \%{ }^{[53]}$, and they are related to functional changes in old age ${ }^{[5,55]}$ but also to intraoperative fluid overload ${ }^{[53]}$. Some conditions such as a lower morbidity rate or a lower intraoperative blood loss in the MILR group might contribute to reduced fluid administration during liver resection. Thus, the absence of large abdominal incision might increase thoracic cage excursion and decrease the pain without respiratory distress. This might be associated with enhanced postoperative recovery and shorter hospital stay.

MILR reduces LOS rate because the absence of large abdominal incision and preservation of postoperative pulmonary function may explain less minor postoperative complications in the MILR group ${ }^{[56]}$. However, careful patient selection about assessment of liver function is the most important factor in morbidity prevention.

This report reveals that operative time in the MILR group was longer than OLR group. The learning curve was associated with experience of surgeons and might be a significant factor contributing to the difference in operative time for the mini-invasive group. The robotic approach, in the MILR group, was associated with longer operative time. This can be explained by the large proportion of major hepatectomy or challenge segment approach, and especially for additional time required for docking and de-docking of robotic system. Tsung et al. ${ }^{[57]}$ found that operative time decreased significantly as the number of cases accumulated and increase of experience with robotic liver surgery.

Oncological outcome such as tumor recurrence and survival did not differ significantly between the two groups, but this outcome was investigated in only half of studies ${ }^{[18,24,25]}$. Recurrence rate is a very important prognostic element. It is essential for improving long-term prognosis, and it is related to tumor-free margins in oncological surgeries, because histologically negative margins could result in a better outcome after HCC resection ${ }^{[38]}$. For patients with HCC, clinical and oncological outcomes are conditioned by tumor invasiveness and underlying liver disease ${ }^{[58]}$. The risk of recurrence of HCC after liver resection is always a concern and is common with the diseased liver remaining in situ. Perhaps not surprisingly, recurrence and survival after surgery for HCC has been shown to be shorter in patients with advanced cirrhosis compared with patients with early disease. The higher recurrence rate during the worsening of the disease probably reflects the carcinogenic effect of advanced cirrhosis, being more prominent than in less cirrhotic livers or in chronic hepatitis, which is well established in the literature ${ }^{[59]}$. Therefore, MILR for HCC provided long-term outcomes that were comparable with OLR and did not generate unusual HCC recurrence patterns.

\section{Study limitation}

There were several limitations in this systematic review. First, the literature search was only done on the two most relevant scientific databases for medical practice (PubMed and Cochrane Library). Second, the review was limited by the lack of randomized controlled studies or prospective studies regarding comparable populations. Indeed most of the studies on this topic were observational and retrospective, although some of them ${ }^{[18,21,23,25]}$ minimized selection bias, performing a matching of the populations studied.

Due to no events in small sample size papers, or outcomes not available in the primary studies, few studies were available in some of the meta-analyses, thus limiting the strength and trustworthiness of our results.

Meta-analyses are characteristically limited by the presence of heterogeneity between studies. Sources of heterogeneity in this review were different patient's age cut-off, different percentages of HCC patients, and 
different countries of studies. We incorporated heterogeneity by performing random effect model metaanalyses. On the contrary, the small numbers of the studies included did not allow us to further explore heterogeneity with subgroup analyses and meta-regression.

Moreover, due to the limited number of the studies included in the quantitative analyses, we were unable to properly verify if publication bias was present. Finally, we observed that our systematic review pooled papers with different study populations. Indeed, two reports included a small number of patients ${ }^{[19,20]}$; however, on the other hand, two main studies analyzed very large populations ${ }^{[21,23]}$.

To conclude, the scientific literature shows the presence of other systematic reviews on this topic. However, all these secondary studies are characterized by different study selection criteria, outcomes, and populations. Consequently, we believe that our paper could add value to the HCC surgical literature, especially because it assessed a very large number of key outcomes.

In conclusion, this study provides an overview of the last ten years about the comparison between MILR and OLR for HCC treatment in elderly patients. Meta-analyses confirmed the advantages of MILR, both laparoscopic and robotic, in terms of perioperative outcomes, where it may promote the extension of liver resection to HCC patients with borderline liver function. Specifically, our results showed shorter LOS, less intraoperative blood loss and lower morbidity rate in MILR. Moreover, major resections were significantly more common in the OLR group compared to the MILR group. There were no significant differences in survival and recurrence outcomes between the two groups.

According to our results, MILR, which minimizes surgical trauma, must be considered as an important treatment option with significant quality of life benefits in the elderly, showing hopefully one of its best advantages in this fragile population. Efforts should be made to avoid as much as possible OLR in this population. However, randomized controlled trials or well-designed large prospective comparative studies would be necessary to definitely support the superiority of MILR in elderly patients with HCC.

\section{DECLARATIONS}

\section{Authors' contributions}

Study conception and design of the work: Brolese A, Ciarleglio FA

Literature search, acquisition, selection and reading: Brolese A, Rigoni M, Vitale A, de Pretis G, Avancini I, Pravadelli C, Frisinghelli M, Rozzanigo U, Luppi G, Dionisi F, Marcucci S, Viel G, Beltempo P, Prezzi C, Frisini M, Brolese M, Nollo G, Ciarleglio FA

Screening of the papers and data extraction from the selected studies: Rigoni M, Ciarleglio FA

Data analysis and statistical evaluation: Rigoni M, Nollo G

Interpretation of data for the work: Brolese A, Rigoni M, Vitale A, Nollo G, Ciarleglio FA

Drafting the work or revising it critically for important intellectual content: Brolese A, Rigoni M, Ciarleglio FA

Final approval of the version to be published: Brolese A, Rigoni M, Nollo G, Ciarleglio FA

\section{Availability of data and materials}

The datasets generated and/or analyzed during the current study are awailable in the cited current literature or websites (PubMed and Cochrane).

\section{Financial support and sponsorship}

None.

\section{Conflicts of interest}

All authors declared that there are no conflicts of interest. 


\section{Ethical approval and consent to participate}

Not applicable.

\section{Consent for publication}

Not applicable.

\section{Copyright}

(c) The Author(s) 2020.

\section{REFERENCES}

1. Benson AB, Abrams TA, Ben-Josef E, Bloomston PM, Botha JF, et al. NCCN clinical practice guidelines in oncology: hepatobiliary cancers. J Natl Compr Cancr Netw 2009;7:350-91.

2. Kabbach G, Assi HA, Bolotin G, Schuster M, Lee HJ, et al. Hepatobiliary tumors: update on diagnosis and management. J Clin Transl Hepatol 2015;3:169-81.

3. Italian Association of Medical Oncologist (AIOM) Guidelines 2019- Hepatocellular Carcinoma (HCC) (in Italian). Avalilable from: https://www.aiom.it/linee-guida-aiom-epatocarcinoma-2019/ [Last accessed on 25 May 2020]

4. Italian Association Of Tumor Registry (AIRTUM) - Cancer Numer in Italy 2019 (in Italian). Available from: https://www.aiom.it/wpcontent/uploads/2019/09/2019_Numeri_Cancro-operatori-web.pdf [Last accessed on 25 May 2020]

5. Italian Association of Medical Oncologist (AIOM) Guidelines 2019 - Cancer in Elderly (in Italian). Avalilable from: https://www.aiom.it/ linee-guida-aiom-tumori-dellanziano-2019/ [Last accessed on 25 May 2020]

6. De Angelis R, Sant M, Coleman MP, Francisci S, Baili P, et al. Cancer Survival in Europe 1999-2007 by country and age: results of EUROCARE -5- a population based study. Lancet Oncol 2014;15:23-34.

7. Yamada S, Shimada M, Miyake H, Utsunomiya T, Morine Y, et al. Outcome of hepatectomy in super-elderly patients with hepatocellular carcinoma. Hepatol Res 2012;42:454-8.

8. Nguyen KT, Marsh JW, Tsung A, Steel JJ, Gamblin TC, et al. Comparative benefits of laparoscopic vs open hepatic resection: a critical appraisal. Arch Surg 2011;146:348-56.

9. Buell JF, Cherqui D, Geller DA, O’Rourke N, Iannitti D, et al. The international position on laparoscopic liver surgery: the Louisville Statement, 2008. Ann Surg 2009;250:825-30.

10. Wakabayashi G, Cherqui D, Geller DA, Buell JF, Kaneko H, et al. Recommendations for laparoscopic liver resection: a report from the second international consensus conference held in Morioka. Ann Surg 2015;261:619-29.

11. Doussot A, Lim C, Lahat E, Salloum C, Osseis M, et al. Complications after hepatectomy for hepatocellular carcinoma independently shorten survival: a western, single-center audit. Ann Surg Oncol 2017;24:1569-78.

12. Nishikawa H, Kimura T, Kita R, Osaki Y. Treatment for hepatocellular carcinoma in elderly patients: a literature review. J Cancer 2013;4:635-43.

13. Moher D, Liberati A, Tetzlaff J, Altman DG, Group P. Preferred reporting items for systematic reviews and meta-analyses: the PRISMA statement. Int J Surg 2010;8:336-41.

14. Clavien PA, Barkun J, de Oliveira ML, Vauthey JN, Dindo D, et al. The Clavien-Dindo classification of surgical complications: five-year experience. Ann Surg 2009;250:187-96.

15. Hozo SP, Djulbegovic B, Hozo I. Estimating the mean and variance from the median, range, and the size of a sample. BMC Med Res Methodol 2005;5:13.

16. Deeks JJ, Higgins JPT, Altman DJ. Analysing data and undertaking meta-analyses. In: Higgins JPT, Green S, editors. Cochrane Handbook for Systematic Reviews of Interventions. Chichester: Wiley \& Sons Ltd; 2008. p. 243-93.

17. DerSimonian R, Laird N. Meta-analysis in clinical trials. Controlled Clinical Trials 1986;7:177-88.

18. Badawy A, Seo S, Toda R, Fuji H, Fukumitsu K, et al. A propensity score-based analysis of laparoscopic liver resection for liver malignancies in elderly patients. J Invest Surg 2017;32:75-82.

19. Chan ACY, Poon RTP, Cheung TT, Chok KSH, Dai WC, et al. Laparoscopic versus open liver resection for elderly patients with malignant liver tumors: a single-center experience. J Gastroenterol Hepatol 2014;29:1279-83.

20. Amato B, Aprea G, De Rosa D, Milone M, di Domenico L, et al. Laparoscopic hepatectomy for HCC in elderly patients: risks and feasibility. Aging Clin Exp Res 2016;29:179-83.

21. Nomi T, Hirokawa F, Kaibori M, Ueno M, Tanaka S, et al. Laparoscopic versus open liver resection for hepatocellular carcinoma in elderly patients: a multi-centre propensity score-based analysis. Surg Endosc 2020;34:658-66.

22. Wang XT, Wang HG, Duan WD, Wu CY, Chen MY, et al. (2015) Pure laparoscopic versus open liver resection for primary liver carcinoma in elderly patients: a single- center, case-matched study. Medicine (Baltimore) 2015;94:e1854.

23. Tee MC, Chen L, Peightal D, Franko J, Kim PT, et al. Minimally invasive hepatectomy is associated with decreased morbidity and resource utilization in the elderly. Surg Endosc 2019; Epub ahead of print. doi: 10.1007/s00464-019-07298-5.

24. Wang WH, Kuo KK, Wang SN, Lee KT. Oncological and surgical result of hepatoma after robot surgery. Surg Endosc 2018;32:3918-24.

25. Chen PD, Wu CY, Hu RH, Chou WH, Lai HS, et al. Robotic versus open hepatectomy for hepatocellular carcinoma: a matched comparison. Ann Surg Oncol 2017;24:1021-8.

26. Hung AK, Guy J. Hepatocellular carcinoma in the elderly: meta-analysis and systematic literature review. World J Gastroenterol 2015;21:12197-210.

27. Chen KW, Ou TM, Hsu CW, Horng CT, Lee CC, et al. Current systemic treatment of hepatocellular carcinoma: a review of the literature. 
World J Hepatol 2015;7:1412-20.

28. Tsujita E, Utsunomiya T, Yamashita Y, Ohta M, Tagawa T, et al. Outcome of hepatectomy in hepatocellular carcinoma patients aged 80 years and older. Hepatogastroenterology 2012;59:1553-5.

29. O’Malley C, Cunningham AJ. Physiologic changes during laparoscopy. Anesthesiol Clin N Am 2001;19:1-19.

30. Demyttenaere S, Feldman LS, Fried GM. Effect of pneumoperitoneum on renal perfusion and function: a systematic review. Surg Endosc 2007;21:152-60.

31. Antoniou SA, Antoniou GA, Koch OO, Pointner R, Granderath FA. Meta-analysis of laparo- scopic vs open cholecystectomy in elderly patients. World J Gastroenterol 2014;20:17626-34.

32. Kunisaki C, Makino H, Takagawa R, Oshima T, Nagano Y, et al. Efficacy of laparoscopy-assisted distal gastrectomy for gastric cancer in the elderly. Surg Endosc 2009;23:377-83.

33. Fujii S, Ishibe A, Ota M, Yamagishi S, Watanabe K, et al. Short-term results of a randomized study between laparoscopic and open surgery in elderly colorectal cancer patients. Surg Endosc 2014;28:466-76.

34. Hinoi T, Kawaguchi Y, Hattori M, Okajima M, Ohdan H, et al.; Japan Society of Laparoscopic Colorectal Surgery. Laparoscopic versus open surgery for colorectal cancer in elderly patients: a multicenter matched case-control study. Ann Surg Oncol 2015;22:2040-50.

35. Fujii S, Tsukamoto M, Fukushima Y, Shimada R, Okamoto K, et al. Systematic review of laparoscopic vs open surgery for colorectal cancer in elderly patients. World J Gastrointest Oncol 2016;8:573-82.

36. Devoto L, Celentano V, Cohen R, Khan J, Chand M. Colorectal cancer surgery in the very elderly patient: a systematic review of laparoscopic versus open colorectal resection. Int J Colorectal Dis 2017;32:1237-42.

37. Yin Z, Fan X, Ye H, Yin D, Wang J. Short and long term outcomes after laparoscopic and open hepatectomy for hepatocellular carcinoma: a global systematic review and meta-analysis. Ann Surg Onc 2013;20:1203-15.

38. Guan R, Chen Y, Yang K, Ma D, Gong X, et al. Clinical efficacy of robot assisted versus laparoscopic liver resection: a meta analysis. Asian J Surg 2019;42:19-31.

39. Bruix J, Sherman M. Management of hepatocellular carcinoma: an update. Hepatology 2011;53:1020-2.

40. Adam R, Frilling A, Elias D, Laurent C, Ramos E, et al. Liver resection of colorectal metastases in elderly patients. Br J Surg 2010;97:366-76.

41. Glassberg MB, Ghosh S, Clymer JW, Wright GWJ, Ferko N, et al. Microwave ablation compared with hepatic resection for the treatment of hepatocellular carcinoma and liver metastases: a systematic review and meta-analysis. World J Surg Oncol 2019;17:98.

42. Xiong JJ, Altaf K, Javed MA, Huang W, Mukherjee R, et al. Meta-analysis of laparoscopic vs open liver resection for hepatocellular carcinoma. World J Gastroenterol 2012;18:6657-68.

43. Takahara T, Wakabayashi G, Beppu T, Aihara A, Hasegawa K, et al. Long-term and perioperative outcomes of laparoscopic versus open liver resection for hepatocellular carcinoma with propensity score matching: a multi-institutional Japanese study. J Hepatobiliary Pancreat Sci 2015;22:721-7.

44. Yoon YI, Kim KH, Kang SH, Kim WJ, Shin MH, et al. Pure laparoscopic versus open right hepatectomy for hepatocellular carcinoma in patients with cirrhosis: a pro- pensity score matched analysis. Ann Surg 2016;265:856-63.

45. Abu Hilal M, Aldrighetti L, Dagher I, Edwin B, Troisi RI, et al. The southampton consensus guidelines for laparoscopic liver surgery: from indication to implementation. Ann Surg 2018;268:11-8.

46. Zhang Y, Huang J, Chen XM, Sun DL. A comparison of laparoscopic versus open left hemihepatectomy for hepatocellular carcinoma. Surg Laparosc Endosc Percutan Tech 2016;26:146-9.

47. Morise Z, Ciria R, Cherqui D, Chen KH, Belli G, et al. Can we expand the indications for laparoscopic liver resection? A systematic review and meta-analysis of laparoscopic liver resection for patients with hepatocellular carcinoma and chronic liver disease. J Hepatobiliary Pancreat Sci 2015;22:342-52.

48. Liu L, Wang Z, Jiang S, Shao B, Liu J, et al. Perioperative allogenenic blood transfusion is associated with worse clinical outcomes for hepatocellular carcinoma: a meta-analysis. PLoS One 2013;8:e64261.

49. Morise Z. Developments and perspectives of laparoscopic liver resection in the treatment of hepatocellular carcinoma. Surg Today 2019;49:649-55.

50. Miyazawa M, Aikawa M, Okada K, Watanabe Y, Okamoto K, et al. Laparoscopic liver resection using a monopolar soft-coagulation device to provide maximum intraoperative bleeding control for the treatment of hepatocellular carcinoma. Surg Endosc 2018;32:2157-8.

51. Levi Sandri GB, Ettorre GM, Aldrighetti L, Cillo U, Dalla Valle R, et al.; I Go MILS Group on HCC. Laparoscopic liver resection of hepatocellular carcinoma located in unfavorable segments: a propensity score-matched analysis from the I Go MILS (Italian Group of Minimally Invasive Liver Surgery) Registry. Surg Endosc 2019;33:1451-8.

52. Nota C, Molenaar IQ, van Hillegersberg R, Borel Rinckes IHM, Hagendoorn J. Robotic liver resection including the posteriosuperior segments:initial experience. J Surg Res 2016;206:133-8.

53. Fuks D, Cauchy F, Ftériche S, Nomi T, Schwarz L, et al. Laparoscopy decreases pulmonary complications in patients undergoing major liver resection: a propensity score analysis. Ann Surg 2016;263:353-61.

54. Lentschener C, Ozier Y. Anaesthesia for elective liver resection: some points should be revisited. Eur J Anaesthesiol 2002;19:780-8.

55. Ford GT, Rosenal TW, Clergue F, Whitelaw WA. Respiratory physiology in upper abdominal surgery. Clin Chest Med 1993;14:237-52.

56. Nanashima A, Abo T, Nonaka T, Fukuoka H, Hidaka S, et al. Prognosis of patients with hepatocellular carcinoma after hepatic resection: are elderly patients suitable for surgery? J Surg Oncol 2011;104:284-91.

57. Tsung A, Geller DA, Sukato DC, Sabbaghian S, Tohme S, et al. Robotic versus laparoscopic hepatectomy: a matched comparison. Ann Surg 2014;259:549-55.

58. Bruix J, Reig M, Sherman M. Evidence-based diagnosis staging, and treatment of patients with hepatocellular carcinoma. Gastroenterology 2016;150:835-53.

59. Taura K, Ikai I, Hatano E, Yasuchika K, Nakajima A, et al. Influence of coexisting cirrhosis on outcomes after partial hepatic resection for hepatocellular carcinoma fulfilling the Milan criteria: an analysis of 293 patients. Surgery 2007;142:685-94. 